\title{
IMPACTO DA RADIAÇÃO NA MODELAGEM DE UMA CALDEIRA DE BAGAÇO DE CANA-DE-AÇÚCAR
}

\author{
D. J. O. FERREIRA ${ }^{1}$, J. H. SOSA ARNAO ${ }^{2}$, B. C. MOREIRA ${ }^{2}$, L. P. RANGEL ${ }^{3}$ e S. W. PARK ${ }^{1}$ \\ ${ }^{1}$ Universidade de São Paulo, Departamento de Engenharia Química \\ ${ }^{2}$ Equipalcool Sistemas, Departamento de Pesquisa e Desenvolvimento \\ ${ }^{3}$ ESSS Engineering Simulation and Scientific Software \\ contato: ${ }^{1}$ chaada@gmail.com, sonwpark@usp.br, ${ }^{2}$ juan@equipalcool.com.br, \\ 3 leonardo@esss.com.br
}

\begin{abstract}
RESUMO - A Fluidodinâmica Computacional (Computational Fluid Dynamics, CFD) tem sido amplamente usada para o desenvolvimento de modelos compreensivos de combustão heterogênea de biomassa. Avalia-se o impacto de diferentes modelos de radiação nos resultados da simulação CFD de uma caldeira de bagaço de cana. Os modelos utilizados, para escoamento turbulento é modelo k- $\varepsilon$ padrão, para a combustão dos gases é EDM (Eddy Dissipation Model) com mecanismo composto de duas reações químicas de combustão do $\mathrm{CH}_{4}$, representando a liberação de hidrocarbonetos leves, e CO. Para o arraste das partículas de bagaço no meio contínuo gasoso é utilizado modelo Schiller-Naumann com os diâmetros de acordo sua análise granulométrica. A radiação foi analisada através de casos simulados sem radiação e a representando pelos métodos P1 e de Transferências Discretas (DTM). A discussão a respeito dos perfis térmicos e de composições químicas dos gases de combustão indicam características e fenômenos internos da fornalha úteis na escolha de condições de operação do equipamento.
\end{abstract}

\section{INTRODUÇÃO}

Enquanto que a troca térmica pelos demais mecanismos é de primeira ordem em relação à diferença de temperatura entre às partes quente e fria, na radiação essa dependência é proporcional à quarta potência da diferença de temperaturas, o que torna seu mecanismo de transferência de energia dominante em reatores nucleares e equipamentos de combustão como fornalhas, turbinas, motores, câmaras de combustão, entre outros (Mbiock e Weber, 2000). A radiação se torna o mecanismo de transferência de calor dominante nos processos em que a fonte térmica excede temperaturas na faixa de 1000 a $1500 \mathrm{~K}$ (Versteeg e Malalasekera, 2007). De acordo com estimativas a partir da metodologia adotada em Sosa-Arnao et al. (2006), no Brasil as caldeiras de queima de bagaço de cana são atingidas, em média, temperaturas de $793 \mathrm{~K}$ para produção de vapor a 6,57 MPa (pressão manométrica) para produção de energia, sendo que na chama são acima de $920 \mathrm{~K}$. Este presente trabalho está dentro de um escopo mais amplo de modelos de combustão em caldeira de bagaço de cana-de-açúcar, e acompanha outro trabalho de Sosa-Arnao et al.(2014). No presente trabalho são realizadas simulações CFD de combustão heterogênea do bagaço de cana de açúcar considerando a troca térmica por radiação no interior da caldeira por Aproximação P1 e por Método das 
Transferências Discretas (DTM, Discrete Transfer Method). Ao desenvolver um modelo CFD para a combustão do bagaço no interior da fornalha de uma caldeira, a troca térmica por radiação deve ser representada adequadamente (Ferreira et al.2012).

\section{MÉTODOS}

O escoamento dos gases de combustão é representado pelo modelo k- $\varepsilon$ padrão por ser adequado a escoamentos industriais confinados com direção preferencial e ser computacionalmente leve em relação a modelos mais avançados como RSM, LES ou DNS. Sua limitação de representação do escoamento nas proximidades da parede é contornada pelo uso de modelo de parede, presente na maioria dos softwares comerciais (Versteeg e Malalasekera, 2007). A combustão em fase gasosa no interior da fornalha é representada pelo modelo EDM (Eddy Dissipation Model), pois é possível considerar que a operação da caldeira bagaço de cana favorece a disponibilidade suficientemente elevada de reagentes e temperaturas para que o processo de queima seja governado pela taxa de mistura dos gases reagentes, determinada pela escala de tempo turbulenta $(\mathrm{k} / \varepsilon$ ou $1 / \omega)$. Para estimar as condições de contorno para a composição dos voláteis inseridos, são utilizadas análises imediata e elementar de amostras de bagaço de cana da região sudeste do Brasil, realizadas pela USP de Ribeirão Preto/SP e Instituto de Pesquisas Tecnológicas de São Paulo (IPT). As espécies consideradas são $\mathrm{CH}_{4}$ (representando todo o conjunto de hidrocarbonetos leves liberados na volatilização), $\mathrm{CO}, \mathrm{H}_{2} \mathrm{O}, \mathrm{CO}_{2} \mathrm{e}$ $\mathrm{H}_{2} \mathrm{O}$. O mecanismo de reação é composto por duas reações químicas: $\mathrm{CH}_{4}+\mathrm{O}_{2} \rightarrow \mathrm{CO}+\mathrm{H}_{2} \mathrm{O}$ e $\mathrm{CO}+$ $\mathrm{O}_{2} \rightarrow \mathrm{CO}_{2}$. Apesar do acoplamento em uma via ser adequado para representar a troca de quantidade de movimento e de massa entre as fases dispersa e contínua por causa da baixa concentração das partículas no domínio computacional, a troca térmica necessita de um acoplamento de duas vias. Se por um lado toda a fonte de combustível provém da volatilização e consumo do carbonizado das partículas de bagaço, por outro, para que tais reações heterogêneas ocorram, é necessário haver fluxos de calor da fase contínua gasosa para as partículas, ativando o processo. Portanto, há uma troca de influências em via dupla entre as fases consideradas. O modelo Schiller-Naumman para o arraste de particulados pode ser considerado adequado para a representação do bagaço de cana porque cada partícula de bagaço deve ser considerada rígida, sem deformação. A distribuição granulométrica das partículas é a utilizada em Sosa-Arnao (2008) para o bagaço de cana obtido após a moenda.

\subsection{Radiação}

A transferência de calor por radiação é descrita na equação de conservação de energia pelo termo de flux térmico $\vec{q}_{r}$ pela equação (1):

$$
\rho c_{P} \frac{D T}{D t}=\beta T \frac{D P}{D t}+\nabla \cdot\left(k \nabla T-\vec{q}_{r}\right)+q^{\prime \prime \prime}+\Phi_{d}
$$

A chamada equação de transferência térmica de radiação espectral (RTE) é descrita pela equação (2) (Vesrteeg e Malalasekera, 2007):

$$
\frac{d I(\vec{r}, \vec{s})}{d s}=\kappa I_{b}(\vec{r})-\kappa I(\vec{r}, \vec{s})-\sigma_{s} I(\vec{r}, \vec{s})+\frac{\sigma_{s}}{4 \pi} \int_{4 \pi} I_{-}\left(\vec{s}_{i}\right) \Phi\left(\vec{s}_{i} \cdot \vec{s}^{\prime}\right) d \Omega_{i}
$$


Em que, para um determinado elemento de meio participante no domínio calculado, o primeiro termo à esquerda da igualdade se refere à varação da intensidade de radiação por comprimento de direção de um feixe, o primeiro termo à direita da igualdade se refere à intensidade radiativa emitida, o segundo termo é a intensidade radiativa absorvida, o terceiro termo representa a intensidade radiativa que sofre espalhamento saindo do elemento e o último termo é referente à intensidade de radiação que entra no elemento também por meio de espalhamento. Ao combinar os termos referentes à absorção e saída por espalhamento da intensidade de radiação, é obtida a variável $\tau$ de acordo com (3) e (4):

$$
\varpi=\frac{\sigma_{s}}{\kappa+\sigma_{s}}, \tau=\int_{0}^{s}\left(\kappa+\sigma_{s}\right) d s^{\prime}
$$

Ao definir uma função termo-fonte dessa variável na equação (5):

$$
S(\tau, \vec{s})=(1-\varpi) I_{b}(\tau)+\frac{\varpi}{4 \pi} \int_{4 \pi} I_{-}\left(\vec{s}_{i}\right) \Phi\left(\vec{s}_{i}, \vec{s}\right) d \Omega_{i}
$$

A equação (2) é simplificada como equação (6):

$$
\frac{d I(\tau, \vec{s})}{d \tau}+I(\tau, \vec{s})=S(\tau, \vec{s})
$$

Devido à natureza complexa, integro-diferencial, da RTE, não existem soluções analíticas para casos com geometrias complexas, havendo a necessidade do uso de modelos numéricos para obter soluções aproximadas. Em ambas metodologias Aproximação P1 e Método das transferências Discretas são adequadas para representação da radiação em equipamentos de combustão confinada como fornalhas e caldeiras em que o meio participante pode ser considerado semi-transparente e a geometria do domínio computacional não apresenta regiões de razão de aspecto elevadas.

A Aproximação P1 expressa o campo de distribuição de intensidade de radiação $I(\vec{r}, \vec{s})=\sum_{l=0}^{\infty} \sum_{m=-l}^{l} I_{l}^{m}(\vec{r}) Y_{l}^{m}(\vec{s})$ por uma série de Fourier 2D truncada no primeiro termo em que a função fluxo térmico intensidade de radiação é representada na equação (7) (Siegel e Howell, 1992):

$$
\vec{q}_{r}=-\frac{1}{3\left(\kappa-\sigma_{s}\right)-A \sigma_{s}} \nabla I_{-}
$$

Apesar do método não apresentar nenhuma restrição quanto à isotropia de emissão, espalhamento ou reflexão da intensidade da radiação, o uso da Aproximação P1 pode apresentar erros significantes em meios "estreitos" (thin) com distribuições de intensidades fortemente anisotrópicas em geometrias 2D e 3D (Lockwood e Shah, 1981). Aproximações de ordem superior, como Aproximação P3, podem minimizar esses problemas mas, no entanto, isso acarretaria em grande esforço computacional e pouco aumento de precisão nos resultados.

Já o método DTM considera a radiação um fenômeno isotrópico quanto à direção e comprimentos de onda do feixes de radiação. Seu conceito básico está na solução do balanço das contribuições da RTE (emissão, absorção, espalhamento e reflexão) em um número previamente definido de direções (paths) que dividem uma esfera ou hemisfério de forma igualitária (isotrópica) 
que unem pares de paredes ou fontes emissoras ou receptoras de radiação. Sendo assim, o método DTM estabelece uma divisão dos hemisférios de um ponto na parede em $\mathrm{N}$ partes em ângulos azimutais, $\delta \theta=\left(\pi / 2 N_{\theta}\right)$, e polares, $\delta \phi=\left(\pi / 2 N_{\phi}\right)$. Para cada parcela do hemisfério divido há um vetor representativo que define um traçado no qual a distribuição da intensidade radiativa em suas contribuições é calculada. A função termo-fonte definida na equação (5) é representada no método DTM pela equação (8) dentro de um somatório de intensidade radiativa média $I_{-, a v e}\left(\vec{s}_{i}\right)$ em:

$$
S(\tau, \vec{s})=(1-\varpi) I_{b}(\tau)+\frac{\varpi}{4 \pi} \sum_{i=1}^{N} I_{-, a v e}\left(\vec{s}_{i}\right) \Phi\left(\vec{s}_{i}, \vec{s}\right) d \Omega
$$

A intensidade inicial de cada feixe em seu elemento de origem na superfície da parede é dada na equação (9) por:

$$
I_{0}=\frac{q_{+}}{\pi}
$$

Em que $q_{+}=\varepsilon_{s} E_{s}+\left(1-\varepsilon_{s}\right) q_{-}$e $q_{-}=\sum_{N} I_{-}(\vec{s}) \vec{s} . \vec{n} d \Omega$. Assim, para o método DTM, o fluxo térmico $\nabla \vec{q}_{r}=\frac{1}{\Delta V} \sum_{k=1}^{N} \delta Q_{g k}$ por radiação pode ser obtido através do cálculo iterativo das equações (10) e (11):

$$
\begin{aligned}
& Q_{s i}=A_{i}\left(q_{+}-q_{-}\right) \\
& \delta Q_{g k}=\int_{\delta \Omega}\left(I_{n+1}-I_{n}\right) A_{i}\left(-\vec{s}_{k} \cdot \vec{n}\right) d \Omega_{k}
\end{aligned}
$$

\section{SIMULAÇÃO}

O domínio computacional representa a fornalha da caldeira de queima de bagaço de cana de açúcar que conta com alimentação de ar primário no piso da caldeira em que seus mais de 26 mil orifícios de entrada em uma grelha são simplificados por 144 superfícies retangulares que representam as placas com grupos de orifícios da grelha móvel. As condições de alimentação de ar secundário, superior nas paredes frontal e traseira e inferior na parede traseira rente à grelha têm seus pontos de injeção representados individualmente. A inserção do bagaço, que ocorre sob rotação devido ao uso de seis espargidores tipo swirl com sentidos alternados é representada através da importação de perfis de velocidade para arranjos de operação para cada um dos alimentadores e 12.000 partículas sob a análise granulométrica do bagaço encontrada em Sosa-Arnao, (2008) são inseridas sob esses perfis de velocidade. A condição de contorno de saída é localizada onde estaria o início dos superaquecedores As paredes do domínio computacional são consideradas sob temperatura constante de $285^{\circ} \mathrm{C}$, temperatura estimada de ebulição da água na pressão de operação fornecida pela Equipalcool Sistemas. A Figura 1 apresenta a visão geral do domínio computacional e a disposição de suas condições de contorno.

São simulados três casos:

- Combustão sem radiação;

- Combustão considerando a radiação representada pela Aproximação P1;

- Combustão considerando a radiação representada por DTM. 

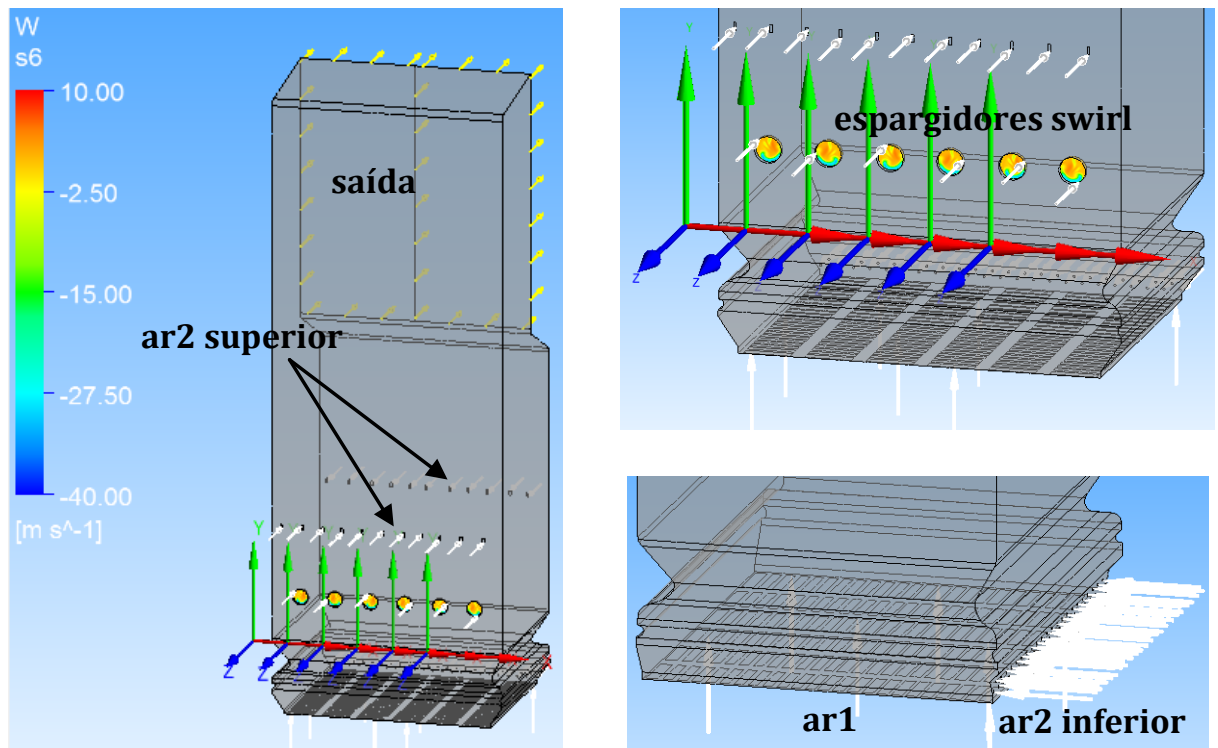

Figura 1: Visão geral das condições de contorno utilizadas na representação da fornalha da caldeira de queima de bagaço de cana de açúcar.

A Tabela 1 apresenta as condições de contorno empregadas nas simulações.

Tabela 1 - Condições de contorno usadas nas simulações da caldeira de bagaço de cana

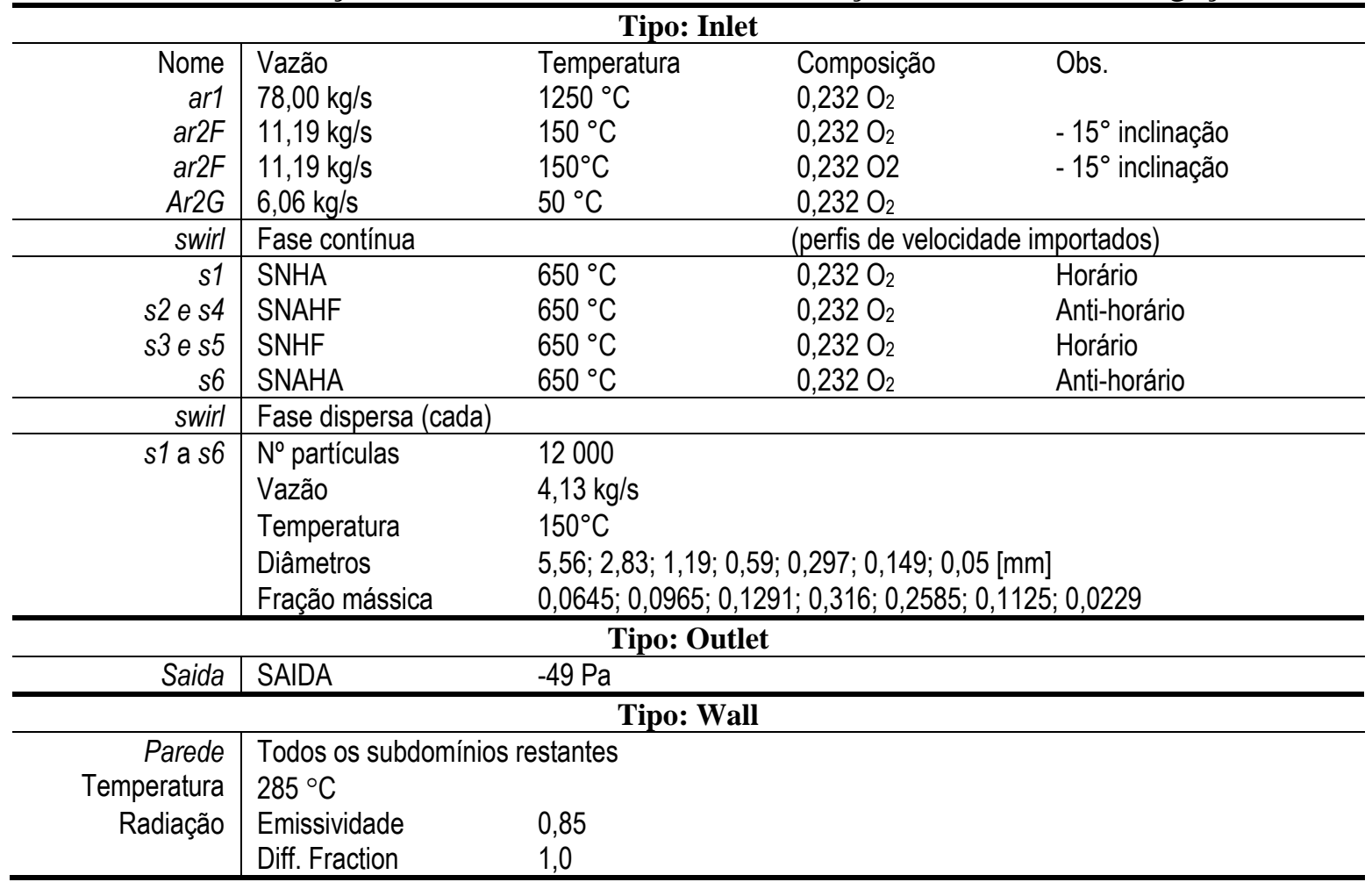


A simulação da caldeira utilizando o método de Aproximação P1 usou os parâmetros padrões considerando espalhamento isotrópico da intensidade radiativa. Para o método DTM, o espalhamento também foi considerado isotrópico, porém foram testadas 8, 10, 12, 16 e 32 direções de feixes de radiação.

\section{RESULTADOS}

Como é esperado que os gradientes de temperatura intensifiquem a turbulência, o escoamento dos gases no interior da fornalha também deve ser afetado pela ausência de radiação. A Figura 2 compara renderizações volumétricas de velocidade dos gases para os três casos simulados.

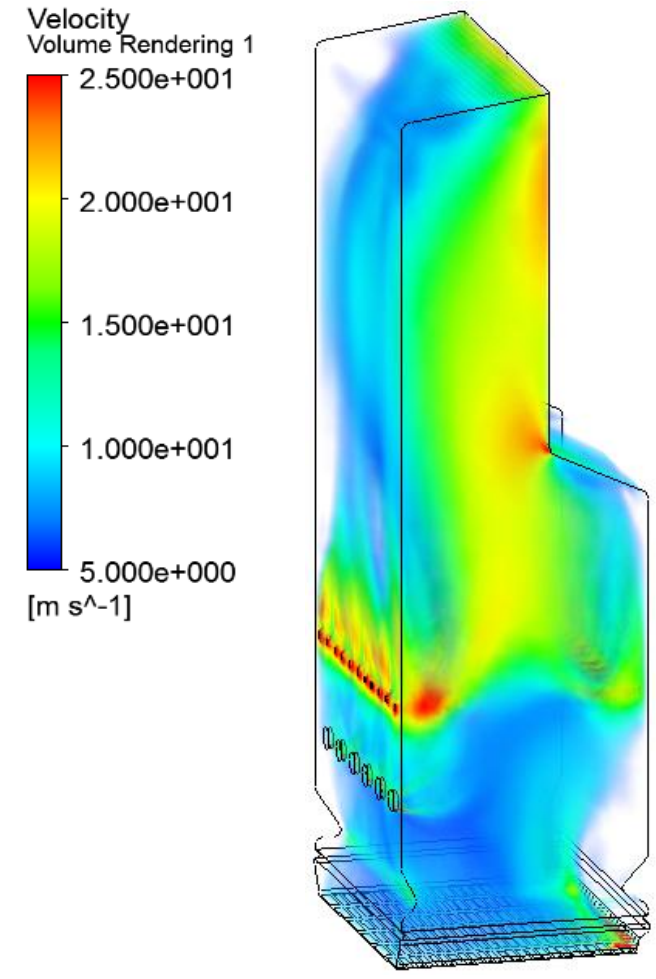

a) Sem radiação

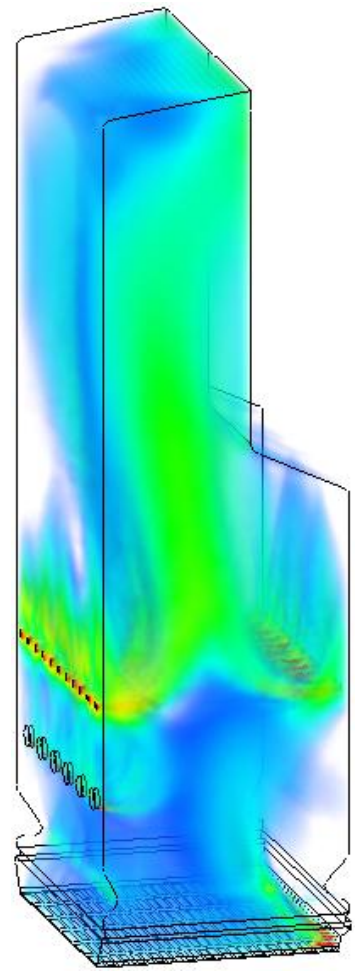

b) Aproximação P1

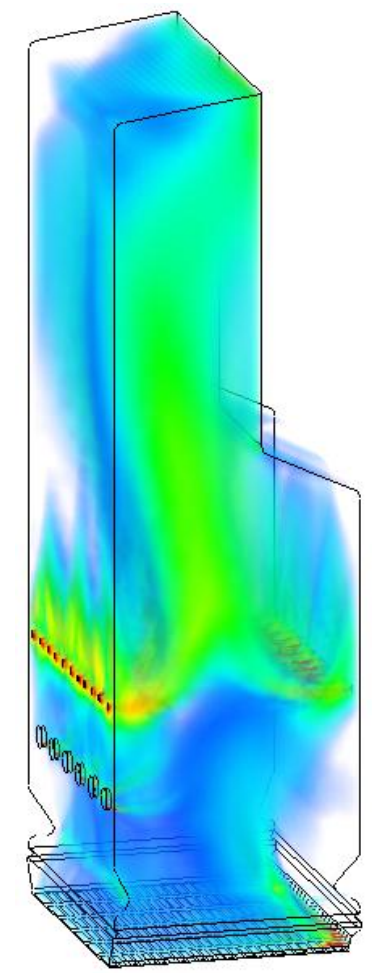

c) DTM

Figura 2: Rederizações volumétricas de velocidade para simulações a) sem a consideração de radiação e com radiação representada por b) Aproximação P1 e c) DTM.

É possível observar na Figura 2 que a consideração de radiação exerce mais influência no escoamento do que as metodologias de representação da radiação em si. Isso ocorre devido ao fato de que a radiação favorece a distribuição de energia na fornalha, pois permite que todos os elementos do domínio computacional exerçam influência uns nos outros. Na ausência de radiação, o fluxo de energia no interior da caldeira fica concentrado onde há escoamento preferencial dos gases de combustão. Sendo assim, é esperado que a convecção seja o mecanismo dominante de transferência térmica, pois a condução entre os gases de combustão e as paredes é limitada à camada limite. A Figura 3 apresenta perfis verticais de temperatura em um plano iso-simétrico (no meio) da fornalha para os três casos simulados. 


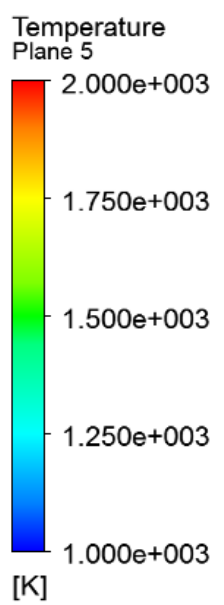

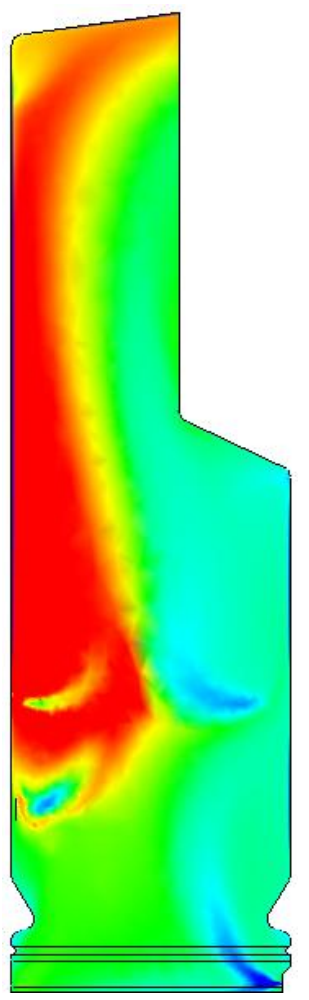

a) Sem radiação

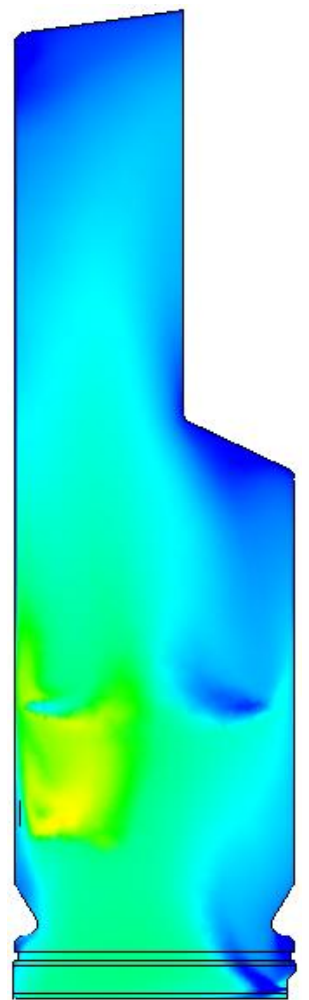

b) Aproximação P1

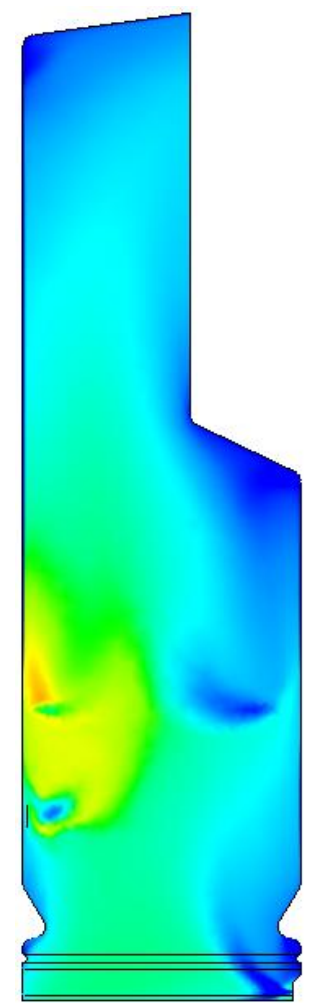

c) DTM

Figura 3: Perfis verticais de temperatura para simulações a) sem a consideração de radiação e com radiação representada por b) Aproximação P1 e c) DTM

Na simulação sem radiação os resultados mostrados na Figura 3 apresentam valores elevados de temperatura nas regiões em que são esperados caminhos preferenciais ascendentes dos gases de combustão e na região superior da fornalha, evidenciando a forte influência da convecção no perfil térmico. Para os casos considerando a radiação, os resultados se mostraram idênticos, o que sugere que, para a faixa de temperatura das simulações e suas respectivas condições de contorno e geometria, ambos métodos são adequados para representação a transferência de calor por radiação no desenvolvimento do modelo de combustão heterogênea do bagaço de cana em caldeira. Nos testes de números de direções dos feixes de intensidade radiativa, os resultados para 8, 10,12, 16 e 32 direções forneceram resultados idênticos, sendo que apenas para 32 direções houve aumento significativo no tempo necessário de processamento para a simulação. Sendo assim, com o objetivo de melhor representar a troca térmica por radiação no interior do equipamento a melhor representação para a radiação é por meio do método DTM com 16 direções, uma vez que o método da Aproximação P1 tende a promover um comportamento numericamente similar a uma segunda difusão térmica, pois apenas adiciona um termo a mais na equação de conservação de energia.

\section{CONCLUSÕES}

A consideração da radiação no modelo demonstrou influência significante no perfil de velocidades. Os perfis de velocidades obtidos para as simulações considerando as duas metodologias 
de representação da radiação se mostraram similares. De acordo com os perfis de temperatura obtidos, a ausência de radiação leva resultados a um comportamento incorreto da operação da caldeira de queima de bagaço de cana de açúcar, pois, a presença de temperaturas elevadas acima do nariz da caldeira acompanhando o escoamento ascendente dos gases é incoerente com o arranjo entrelaçado das portas de ar secundário superior, a alimentação do ar primário em grelhas, a inserção do bagaço sob forte mistura e rotação e o próprio formato da caldeira que visão manter a combustão do bagaço abaixo do ar secundário superior. Portanto, para que o perfil térmico seja preciso em relação às suas estimativas é necessário que o modelo CFD considere adequadamente a transferência térmica por radiação para a queima de bagaço de cana em caldeira.

\section{AGRADECIMENTOS}

Autores agradecem a FAPESP (São Paulo) pelos recursos do Projeto 2010/50389-3 "Aplicação da Fluidodinâmica Computacional a uma Caldeira de Bagaço; e pelo auxílio viagem para participação no presente CoBEQ 2014.

\section{REFERÊNCIAS}

Ferreira, D.J.O.; Cardoso, M.; Park, S.W.; The impact of radiation on gas combustion modeling for a kraft recovery boiler. Proceedings of 11th International Symposium on Process Systems Engineering, Singapore, 15-19 July 2012.

Lockwood, F.C.; Shah, N.G.; A new radiation solution method for incorporation in general combustion prediction procedures. Eighteenth Symposium (International) on Combustion. pp. 14051414,1981

Mbiock, A.; Weber, R.; Radiations in Enclosures - Elliptic Boundary Value Problem. SpringerVerlag Berlin Heidelberg, 2000.

Siegel, R.; Howell, R.; Thermal Radiation Heat Transfer. Third Edition. Taylor \& Francis Inc., 1992.

Sosa-Arnao, J.H.; Modesto, M.; Nebra, S.A.; Two proposals to determine the efficiency of bagasse boiler. In Proceedings of the 6. Encontro de Energia no Meio Rural. 2006.

Sosa-Arnao, J.H.; Caldeiras aquatubulares de bagaço - Estudo do sistema de recuperação de energia. Tese de doutorado, UNICAMP, Campinas, SP, 2008

Sosa-Arnao, J. H.; Ferreira, D.J.O.; Santos, C.G ; Alvarez-Jácobo, J.E.; Rangel, L.P.; Park, S.W. A Influência da geometria do espargidor na injeção e queima de bagaço de cana-de-açúcar. $C O B E Q$ 2014 - XX Congresso Brasileiro de Engenharia Química. Florianópolis, Brasil. 19-22 de Outubro de 2014.

Versteeg, H.K.; Malalasekera, W.; An introduction to computational fluid dynamics - the finite volume method. Second edition. England, Pearson Education Limited, 2007. 